\title{
Magnetization of silicene via coverage with gadolinium: Effects of thickness, symmetry, strain, and coverage
}

\author{
Salih Demirci®, ${ }^{1}$ Taylan Gorkan, ${ }^{2}$ Şafak Çallioğlu $\odot,{ }^{3}$ Yusuf Yüksel $\odot,{ }^{4}$ Ümit Akıncı, ${ }^{4}$ \\ Ethem Aktürk $\odot,^{2,5, *}$ and Salim Ciraci ${ }^{6, \dagger}$ \\ ${ }^{1}$ Department of Physics, Kırlkkale University, Kırıkkale 71450, Turkey \\ ${ }^{2}$ Department of Physics, Adnan Menderes University, 09100 Aydin, Turkey \\ ${ }^{3}$ Department of Electrical and Electronic Engineering, Bilkent University, Ankara 06800, Turkey \\ ${ }^{4}$ Dokuz Eylul University, Faculty of Science, Physics Department, Tinaztepe Campus, 35390 Izmir, Turkey \\ ${ }^{5}$ Physik Department E20, Technische Universität München, 85748 Garching, Germany \\ ${ }^{6}$ Department of Physics, Bilkent University, Ankara 06800, Turkey
}

(Received 27 September 2021; accepted 14 December 2021; published 23 December 2021)

\begin{abstract}
When covered by gadolinium (Gd) atoms, silicene, a freestanding monolayer of Si atoms in a honeycomb network, remains stable above the room temperature and becomes a two-dimensional (2D) ferromagnetic semiconductor, despite the antiferromagnetic ground state of three-dimensional bulk $\mathrm{GdSi}_{2}$ crystal. In thin $\mathrm{GdSi}_{2}$ multilayers, even if magnetic moments are ordered parallel in the same Gd atomic planes, they are antiparallel between nearest Gd planes; hence they exhibit a ferrimagnetic behavior. In contrast, a freestanding $\mathrm{Gd}_{2} \mathrm{Si}_{2}$ monolayer constructed by covering silicene from both sides by $\mathrm{Gd}$ atoms is a stable antiferromagnetic metal due to the mirror symmetry. While multilayers covered by Gd from both sides having an odd number of Gd planes have a ferrimagneticlike ground state, even-numbered ones have antiferromagnetic ground state, but none of them is ferromagnetic. Silicon atoms intervening between Gd planes are responsible for these intriguing magnetic orders conforming with the recent experiments performed on $\mathrm{Si}(111)$ surface. Additionally, the magnetic states of these 2D gadolinium disilicide monolayers can be monitored by applied tensile strain and by the coverage/decoration of Gd. These predictions obtained by using first-principles, spin-polarized, density functional theory calculations combined with Monte Carlo simulations herald that $\mathrm{C}, \mathrm{B}, \mathrm{Si}, \mathrm{Ge}, \mathrm{Sn}$, and their compounds functionalized by rare-earth atoms can lead to novel nanostructures in $2 \mathrm{D}$ spintronics.
\end{abstract}

DOI: 10.1103/PhysRevB.104.224427

\section{INTRODUCTION}

Functionalization of bare two-dimensional (2D) nanostructures through the chemisorption of adatoms has developed as a powerful theoretical and experimental tool to construct novel nanomaterials with unusual properties. While the realization of long-range magnetic order in $2 \mathrm{D}$ systems was known to be suppressed by thermal fluctuations [1], 2D monolayers (like graphene, silicene, and germanene, i.e., group-IV elements in a honeycomb network) were able to attain intrinsic magnetism, when they covered lanthanide atoms adsorbed to the (111) surfaces of their parent crystals to construct structures such as $\mathrm{GdSi}_{2}$ and $\mathrm{GdGe}_{2}$ overlayers [2-4]. Furthermore, the magnetic ground state of these functionalized monolayers has changed in their thin multilayers and also in their parent, three-dimensional (3D) bulk crystals [2-4]. Functionalization of graphene [5] to have intrinsic ferromagnetic state is highly desired in graphene-based spintronics. Similarly, silicene and germanene, which were first predicted theoretically [6-9] and later synthesized [10,11], keep the promises of novel nanoelectronic applications because of their parent crystals

\footnotetext{
*ethem.aktuerk@tum.de

†ciraci@fen.bilkent.edu.tr
}

with well-developed technologies in microelectronics. Functionalization of these $2 \mathrm{D}$ materials to acquire an intrinsic ferromagnetism is of critical importance in nanospintronics. As a matter of fact, not only these three honeycomb structures, but also $2 \mathrm{D} \mathrm{Gd}_{2} \mathrm{~B}_{2}$ monolayer derived from $\mathrm{GdB}_{2}$ layers of $3 \mathrm{D}$ bulk, a member of the hexagonal rare-earth metal diboride family conforming $P 6 / \mathrm{mmm}$ space group symmetry, acquired above room temperature magnetism following its functionalization by the coverage of $\mathrm{Gd}$ atoms to its bare side [12]. In this respect, a theoretical analysis of adsorption of lanthanide atoms on silicene and the resulting magnetic state open ways to achieve similar type magnetization in other 2D monolayers, such as other III-V compounds [9], pnictogens [13], and transition metal dichalcogenides.

Magnetism in 2D monolayers, by itself, is an emerging field of fundamental importance, despite some issues are still unknown. Magnetic states of 2D monolayers have been brought into focus [14-16] through the recent observations on 2D van der Waals (vdW) structures [17,18], $\mathrm{CrI}_{3}$ and $\mathrm{CrGeTe}_{3}$. The ferromagnetic (FM) ground state of bulk (multilayer) $\mathrm{CrI}_{3}$ displays a crucial dependence on the thickness. For instance, while its bilayer acquires an antiferromagnetic (AFM) ground state, the monolayer changes to become FM again [17]. Even more remarkable is that the $\mathrm{CrI}_{3}$ bilayer at fixed magnetic fields near the metamagnetic transition can 
switch from AFM state to FM state with applied gate voltage offering electrically tunable magnetism [19]. Clearly, switching the magnetic state of a $2 \mathrm{D}$ material by external agents is now a rapidly developing field of research. Understanding the physical mechanism underlying intrinsic magnetism and its variation with thickness, applied strain, and pressure, applied electric and magnetic fields are fundamental and can provide for useful new effects to control the magnetic state of the structure.

This theoretical study investigates the magnetization of silicene by the coverage of $\mathrm{Gd}$ atoms, a rare-earth element, to form a freestanding $2 \mathrm{D} \mathrm{GdSi}{ }_{2}$ monolayer and the interplay of the magnetic ground states in their multilayers (obtained by asymmetric and symmetric stacking of monolayers) and eventually in 3D layered $\mathrm{GdSi}_{2}$, gadolinium silicide crystal. The latter 3D crystal has already been a focus of interest two decades ago in improving the parasitic problems emerging as the device sizes (speeds) become smaller (faster) in microelectronics [20]. Here, we have focused on the freestanding, 2D $\mathrm{GdSi}_{2}$ monolayer. Having assured the dynamical and thermal stability of this monolayer, we examined the nature of the binding between Gd overlayer and silicene, and the charge redistribution between them. Our study based on first-principles density functional theory (DFT) predicts that the freestanding $2 \mathrm{D} \mathrm{GdSi}_{2}$ monolayer is an FM semiconductor having different band gaps for different spin direction. Furthermore, in order to understand how the AFM state of the $3 \mathrm{D}$ bulk $\mathrm{GdSi}_{2}$ switches to the FM state in $2 \mathrm{D} \mathrm{GdSi}{ }_{2}$ monolayer as observed experimentally [2] and what the character of exchange interactions is, we studied the magnetic states of the bilayer and multilayers (namely, 3L, 4L). To reveal the effect of symmetry of the atomic structure perpendicular to the layers, we also considered the freestanding silicene covered with $\mathrm{Gd}$ adatoms at both sides to construct $\mathrm{Gd}_{2} \mathrm{Si}_{2}$, a new 2D monolayer. Furthermore, we examined the bilayer and multilayers of the 2D $\mathrm{GdSi}_{2}$ monolayer, saturated with $\mathrm{Gd}$ atoms from both sides like $2 \mathrm{D} \mathrm{Gd}_{2} \mathrm{Si}_{2}$ monolayer. Using the exchange coupling and magnetic anisotropy constants extracted from the firstprinciples calculations, we performed classical Monte Carlo calculations and revealed the magnetic and thermodynamic properties of monolayers and their critical temperatures.

The variation of the magnetism with various external effects is an important aspect of the present study. Within this context, we examined the effects of 2D strain on the monolayers and discussed the coverage (decoration) of Gd-like $(1 \times 2)$ to construct one-dimensional (1D) rods (nanowires) on silicene. The amazing interplay of the ground states among FM, AFM, and ferrimagnetic states with coverage, stacking symmetry, and layer number has been investigated and the effect of nonmagnetic silicene in the intriguing magnetic interactions has been unveiled. Present results suggest efficient ways of monitoring 2D magnetism for spintronic applications.

\section{COMPUTATIONAL DETAILS}

We performed our theoretical analysis within the firstprinciples plane-wave calculations based on spin-polarized DFT by using projected augmented wave [21,22] potentials to describe the ion-electron interaction, as implemented in the Vienna $A b$ initio Simulation Package, VASP [23].
For the exchange-correlation potential, generalized gradient approximation was adopted by using the Perdew-BurkeErnzerhof (PBE) [24] functional. On-site Coulomb interaction [25] has been treated by Hubbard $U$ correction with interaction parameters of $U=6.7 \mathrm{eV}$ and $J=0.7 \mathrm{eV}$ [26-28]. All structures in this study have been treated by including spinorbit coupling (SOC) in these $\mathrm{PBE}+U$ calculations identified as $\mathrm{PBE}+U+\mathrm{SOC}$ throughout the text. The vdW correction was taken into account using the method of Grimme (DFT-D2) [29]. Brillouin zone (BZ) integration is performed with automatically generated $16 \times 16 \times 1 k$-point grid in the Monkhorst-Pack scheme [30]. The energy cutoff of the planewave basis was used as $340 \mathrm{eV}$. The geometry optimizations including lattice constant relaxation were achieved by using conjugate-gradient algorithm [31,32] until the total energy and forces were minimized and the maximum pressure in the unit cell was reduced to less than $0.5 \mathrm{kbar}$. The energy convergence value was adjusted to be $10^{-5} \mathrm{eV}$ between two successive electronic steps and the force on each atom is less than $10^{-2} \mathrm{eV} / \AA$.

The dynamical stability of all structures is assured by the calculations of frequencies of phonon modes with $\omega(\mathbf{k})^{2} \geqslant 0$. In order to compute the phonon modes, we calculated force constants of the $(3 \times 3 \times 1)$ supercell in real space using density functional perturbation theory [33] as implemented in the PHONOPY [34] program. The thermal stability of these systems is tested by $a b$ initio molecular dynamics calculations at finite temperature. The time step between ionic iterations was taken to be $2 f s$. Every 50 steps velocities are rescaled to match the desired temperature. All the structures were visualized in the VESTA code [35].

In addition to $\mathrm{PBE}+U+\mathrm{SOC}$ calculations, we also applied the Heyd-Scuseria-Ernzerhof (HSE06) hybrid functionals method [36] to PBE results in order to obtain corrected energy band gap values. It is also known that HSE06 calculations were able to give realistic magnetic ground states and their magnetic moments [37]. In our study the HSE06 functional is constructed by mixing $25 \%$ of the Fock exchange with $75 \%$ of the PBE exchange and $100 \%$ of the PBE correlation.

\section{ELECTRONIC AND MAGNETIC PROPERTIES OF GdSi}

In this section, we discuss electronic and magnetic properties of the freestanding, $2 \mathrm{D} \mathrm{GdSi} \mathrm{i}_{2}$ monolayer, its bilayer and multilayers, as well as $3 \mathrm{D} \mathrm{GdSi}_{2}$ bulk crystal calculated within $\mathrm{PBE}+U+\mathrm{SOC}$ and HSE as described in Sec. II.

\section{A. Electronic and magnetic properties of 2D monolayer}

Experimentally, Tokmachev et al. [2] synthesized $\mathrm{GdSi}_{2}$ monolayer on $\mathrm{Si}(111)$ surface by molecular beam epitaxy. Here, we consider freestanding, $2 \mathrm{D} \mathrm{GdSi}{ }_{2}$ monolayer (i.e., a freestanding silicene monolayer in honeycomb network, which is fully covered by Gd atoms adsorbed above the center of each hexagon of $\mathrm{Si}$ atoms). This adsorption site of $\mathrm{Gd}$ atoms, named as hollow site, is most favorable energetically. In Fig. 1(a), the optimized atomic structure and its relevant structural parameters are shown. Notably, the buckling of 

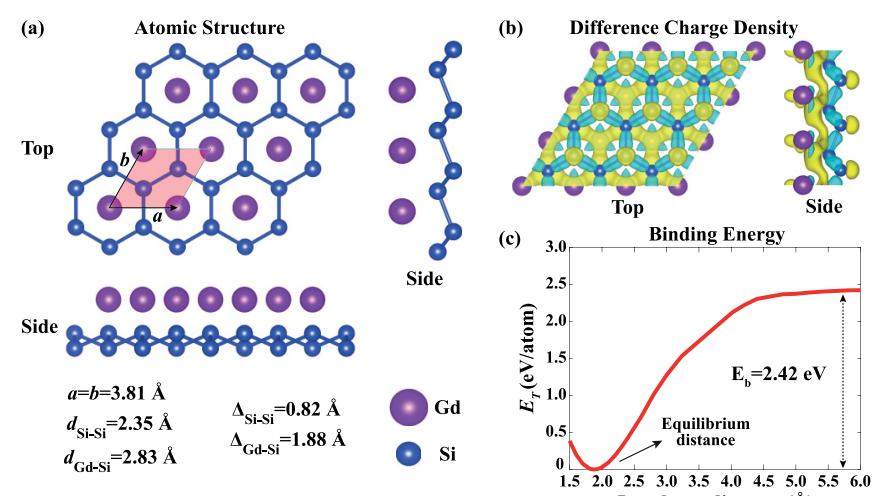

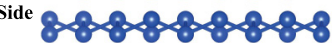

$$
\begin{array}{ccc}
a=b=3.81 \AA & \Delta_{\mathrm{Si} S \mathrm{~S}}=0.82 \AA & \odot \mathrm{Gd} \\
d_{\mathrm{Si}-\mathrm{S}}=2.35 \AA & \Delta_{\mathrm{Gd}-\mathrm{Si}}=1.88 \AA & \mathrm{si}^{2} \\
d_{\mathrm{Gd}-\mathrm{Si}}=2.83 \AA & &
\end{array}
$$

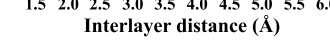

FIG. 1. GdSi 2 monolayer: (a) Top and side views (from two different directions) of the optimized atomic structure with calculated relevant structural parameters. $\mathrm{Gd}(\mathrm{Si})$ atoms are shown by large/purple (small/blue) balls. The unit cell is shaded. The buckling of silicene, $\Delta_{\mathrm{Si} \text {-Si }}$ has decreased upon the coverage of $\mathrm{Gd}$ atoms. (b) Top and side views of the isosurfaces of the difference charge density, $\Delta \rho(\mathbf{r})$ (see text). Accumulation (depletion) of the electronic charge is shown in yellow (turquoise). (c) Variation of the total energy, $E_{T}$, with the minimum distance, $d$, between Gd overlayer and silicene, and the binding energy, $E_{b}$, in equilibrium. The zero of the total energy is set at the minimum.

silicene, even if reduced from its bare value, continues to exist after the coverage with Gd atoms. In Fig. 1(b), the difference charge density, $\Delta \rho(\mathbf{r})$, which is obtained by subtracting the charge density of Gd overlayer and bare silicene from that of $2 \mathrm{D} \mathrm{GdSi}_{2}$ monolayer shows charge accumulation between overlayer and silicene, and also at its uncovered surface. In Fig. 1(c), the variation of the total energy with the distance of $\mathrm{Gd}$ overlayer from the plane of upraised $\mathrm{Si}$ atoms, $d$, is presented. The equilibrium binding energy of the Gd overlayer is estimated to be $2.42 \mathrm{eV}$ per $\mathrm{Gd}$ atom, which is rather strong and confirms charge exchange between $\mathrm{Gd}$ overlayer and silicene.

The dynamical stability test is realized by the calculations of frequencies of phonon modes with $\omega(\mathbf{k})^{2} \geqslant 0$. In Fig. 2(a), phonon bands, i.e., $\omega(\mathbf{k})$, are presented. The thermal stability tests realized by $a b$ initio molecular dynamics calculations were performed at $T=1000 \mathrm{~K}$ for $2 \mathrm{ps}$; during this time period $\mathrm{GdSi}_{2}$ monolayer remained stable. Electronic structure calculations for the optimized structure using $\mathrm{PBE}+U+$ SOC indicate that $2 \mathrm{D} \mathrm{GdSi}_{2}$ monolayer has an FM ground state with a total magnetic moment $\mu_{T}=7.94 \mu_{B}$ per unit cell (7.32 $\mu_{B}$ of it is located at Gd atoms). Hence, the majority of this magnetic moment is located on $\mathrm{Gd}$ atoms and originates from its $4 f$ orbitals.

The electronic energy bands of $2 \mathrm{D} \mathrm{GdSi}_{2}$ calculated using $\mathrm{PBE}+U+\mathrm{SOC}$ presented in Fig. 2(b) depict a very narrow band gap along the $M-K$ direction of the hexagonal Brillouin zone. The calculated components of the total magnetic moment are $\mu_{T, x}=4.58 \mu_{B}, \mu_{T, y}=4.58 \mu_{B}$, and $\mu_{T, z}=$ $4.58 \mu_{B}$ per unit cell with a magnitude $\mu_{T}=7.94 \mu_{B}$ per unit cell (7.32 $\mu_{B}$ of it is located at Gd atoms). Specifying the total energies with magnetization along the direction $[h, k, l]$ as $E_{T,[h k l]}, E_{T,[100]}=E_{T,[010]}=E_{o}=-24.734 \mathrm{eV}$ corresponds to the most stable spin orientation (or easy axis). Then, the
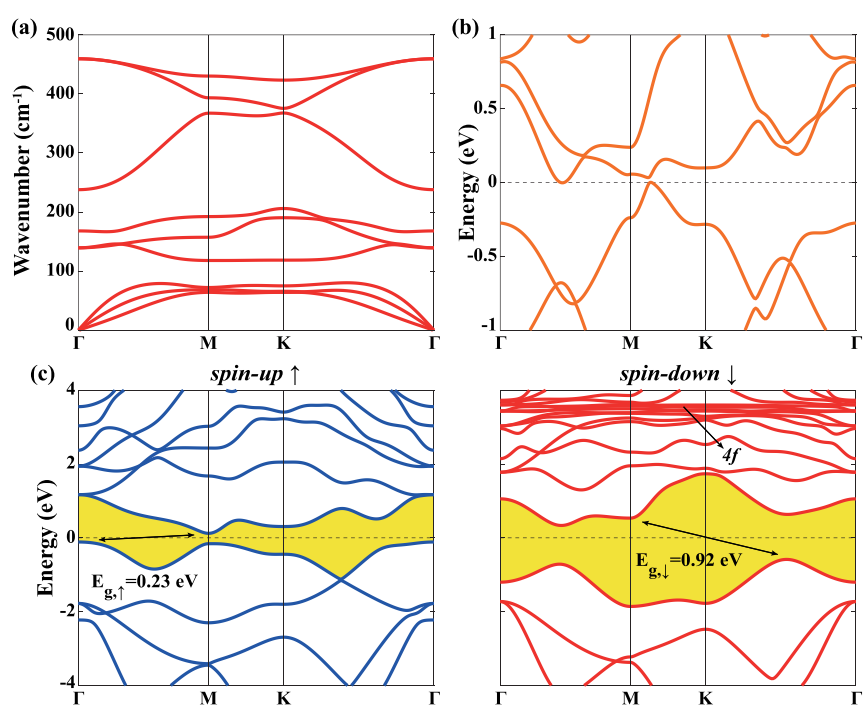

FIG. 2. 2D GdSi ${ }_{2}$ monolayer: (a) Phonon bands along relevant symmetry direction of the hexagonal Brillouin zone. (b) Electronic energy band structure calculated by $\mathrm{PBE}+U+\mathrm{SOC}$ (with Hubbard $U=6.7 \mathrm{eV}$ and $J=0.7 \mathrm{eV}$ ). (c) Electronic energy band structure of spin-up $(\uparrow)$ and spin-down states $(\downarrow)$ and corresponding band gaps $E_{g, \uparrow}$ and $E_{g, \downarrow}$ obtained from HSE calculations. Zero of energy is set at the Fermi level.

magnetocrystalline anisotropy energies for a specific direction are calculated as $E_{M A,[h k l]}=E_{T,[h k l]}-E_{o}$. Here we calculated $E_{M A,[001]}=223 \mu \mathrm{eV}, E_{M A,[101]}=109 \mu \mathrm{eV}$, and $E_{M A,[111]}=$ $72 \mu \mathrm{eV}$.

Our calculations using the HSE method, which is known to give realistic energy band gaps between valence and conduction bands, predict that $2 \mathrm{D} \mathrm{GdSi}_{2}$ monolayer is an FM semiconductor with the total magnetic moment of $\mu_{T}=$ $8.0 \mu_{B}$ per unit cell. Its band gap for spin-up electrons is $E_{g, \uparrow}=0.23 \mathrm{eV}$ and for spin-down electrons, $E_{g, \downarrow}=0.92 \mathrm{eV}$. Figure 2(c) presents spin-polarized electronic energy bands calculated by using HSE. One can deduce how the band gap is opened by comparing the bands in Figs. 2(b) and 2(c). The analysis of the orbital projected density of states demonstrates that the binding of Gd atoms to silicene is provided mainly by $\mathrm{Si} p$ orbitals and $\mathrm{Gd} p, d$ orbitals, whereas the contribution of Gd $4 f$ orbitals to the binding is minute. Flat bands derived from $\mathrm{Gd} 4 f$ orbitals appear at $\sim-8.7 \mathrm{eV}$ in the valence band and $\sim 3.5 \mathrm{eV}$ in the conduction band. Accordingly, $\mathrm{Gd}$ atoms are bound to silicene through their outer valence orbitals and attribute ferromagnetism to the monolayer through their inner $4 f$ orbitals. Since $\mathrm{Gd} 4 f$ orbitals in $2 \mathrm{D} \mathrm{GdSi}_{2}$ monolayer are almost intact, the calculated magnetic moment located at each adsorbed $\mathrm{Gd}$ atom complies with the effective Bohr magneton of $\mathrm{Gd}^{+3}$ ions, $p=g[j(J+1)]^{1 / 2}$ with $J$ obtained according to Hund's rule. The above results conform with the experimental data [2] assigning an intrinsic FM ground state to $\mathrm{GdSi}_{2}$ grown on $\mathrm{Si}(111)$ surface.

In order to determine the magnetic constants necessary for Monte Carlo simulations, whereby the magnetic ground state can be obtained with higher degrees of freedom and relevant magnetic/thermodynamic properties can be revealed, we also calculated the total energies corresponding to a rectangular 


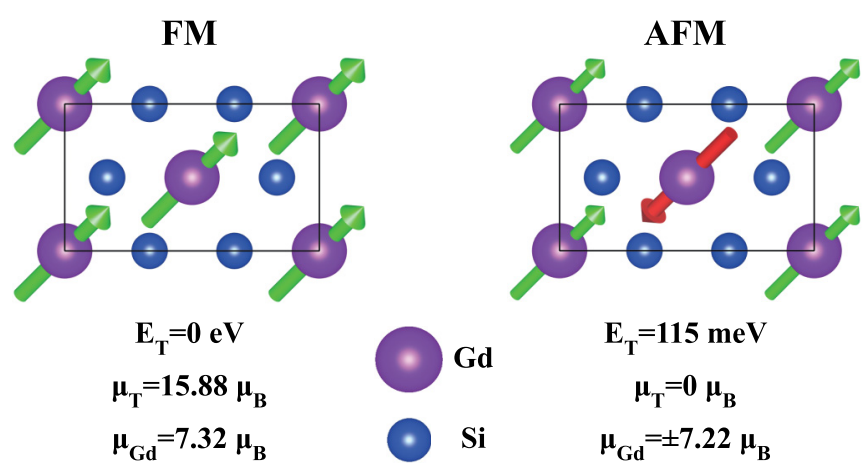

FIG. 3. Top and side views of the atomic structure of $2 \mathrm{D} \mathrm{GdSi} i_{2}$ monolayer in a rectangular supercell including $2 \mathrm{Gd}$ and $4 \mathrm{Si}$ atoms. Two different spin configurations, FM and AFM, are described in top views. The calculated total magnetic moment and the magnetic moment at each Gd atom are given for FM and AFM configurations.

cell of $\mathrm{GdSi}_{2}$ monolayer comprising $2 \mathrm{Gd}$ and $4 \mathrm{Si}$ atoms and allowing FM and AFM magnetic configurations. In Fig. 3, we show FM and AFM configurations in this rectangular cell, where the FM configuration is $115 \mathrm{meV}$ lower energy (favorable) per rectangular cell to the AFM configuration. Monte Carlo simulations presented in the forthcoming section confirm the FM ground state of the $2 \mathrm{D} \mathrm{GdSi}_{2}$ monolayer.

\section{B. 2D bilayer and multilayers}

Switching of the AFM ground state of 3D bulk $\mathrm{GdSi}_{2}$ to FM in 2D monolayer is of fundamental interest. Similar changes have been observed recently also in different 2D structures [14-18]. Here, starting from the bilayer, we investigate how the magnetic ground state changes in multilayers. To this end, we consider the bilayer (2L), $3 \mathrm{~L}$, and $4 \mathrm{~L}$ of $\mathrm{GdSi}_{2}$, where monolayers are stacked one above the other with atoms occupying similar lateral positions. The spacing between the layers and atomic positions are determined after full structure optimization by the minimization of the total energy including the vdW interaction [29]. These multilayers are beyond $\mathrm{vdW}$ solids because of the significant chemical interlayer interaction. The absence of mirror symmetry along the stacking direction, which is perpendicular to layers, shows effects in the atomic structure, as well as in magnetic properties. For example, the buckling of silicene, which is 0.83 $\AA$ in the monolayer, changes to $0.71 \AA$ for the top and to $0.20 \AA$ for the bottom layer of the bilayer. The calculated values of buckling in $3 \mathrm{~L}, 0.73 \AA$ for the top, $0.39 \AA$ for the middle, and $0.24 \AA$ for the bottom layer. Also, similar variations in the distances between consecutive Gd overlayers occur due to asymmetry in stacking.

The magnetic moments of $\mathrm{Gd}$ atoms in different layers and also the resulting magnetic ground states of $2 \mathrm{D} \mathrm{GdSi}$ multilayers vary with the number of $\mathrm{GdSi}_{2}$ monolayers. As an example, since the magnetic moments of $\mathrm{Gd}$ atoms are different in different layers of the bilayer, the AFM ground state does not occur even if magnetic moments of adjacent layers favor antiparallel alignment. Due to this effect, the total magnetic moment of the bilayer is rather small; only $0.38 \mu_{B}$ per cell, but not zero. This configuration, which is named as ferrimagnetic (FerriM), has the lowest total energy for the bi-

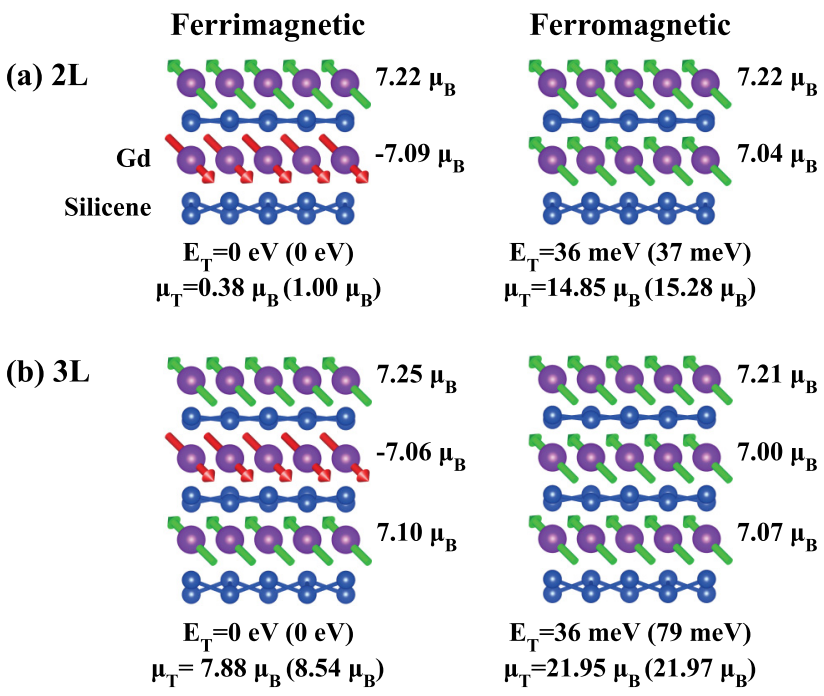

(c) $4 \mathrm{~L}$
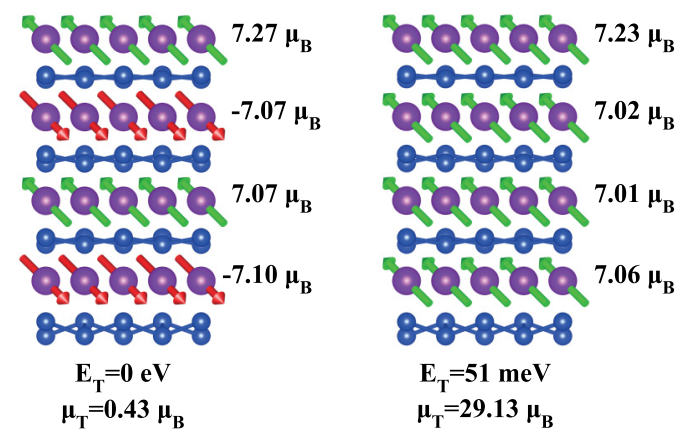

\section{Anti-Ferrimagnetic}

(d) Bulk

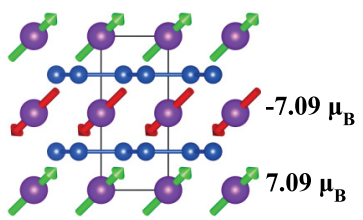

\section{Ferromagnetic}

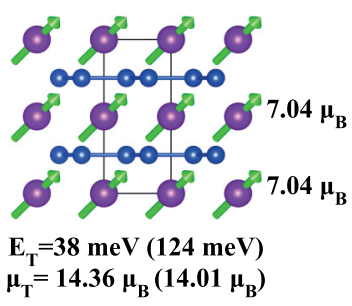

FIG. 4. Side views of the atomic structure of multilayers formed by the stacking of $2 \mathrm{D} \mathrm{GdSi}_{2}$ monolayers. (a) Bilayer (2L) with FerriM ground and FM excited state. Calculated total energies $E_{T}$ and magnetic moments $\mu_{T}$ per cell are indicated for each multilayer. Values in parentheses are obtained from HSE calculations and are given for comparison. (b) Same as (a) for 3L. (c) 4L. (d) 3D bulk in hexagonal structure with AFM ground and FM exited states, and corresponding total energies and magnetic moments per cell.

layer. In contrast, the FM state, where the magnetic moments of Gd atoms in different layers are parallel to give $14.85 \mu_{B}$ is not a ground state for the bilayer. These interesting variations are schematically described in Fig. 4 for $2 \mathrm{~L}, 3 \mathrm{~L}$, and $4 \mathrm{~L}$. Like bilayer, owing to the slightly different magnetic moments of $\mathrm{Gd}$ atoms at different layers and exchange interaction favoring antiparallel spin orientation at $\mathrm{Gd}$ atoms in adjacent layers, $\mathrm{GdSi}_{2}$, the multilayers with even number of layers $(4 \mathrm{~L})$ have small magnetic moments $\left(\leqslant 0.5 \mu_{B}\right.$ per cell). In contrast, the multilayers with odd number of layers, for example $3 \mathrm{~L}$, have significant magnetic moments (like $\sim 7 \mu_{B}$ per cell). 
The variation of the total/net magnetic moment of $\mathrm{GdSi}_{2}$ multilayers with their number of layers despite their finite thickness emerges from the asymmetric stacking of layers and gives rise to an even-odd disparity attributing a kind of FerriM ground state. We note that calculations with larger cells allowing AFM configuration in the same layer, but parallel or antiparallel spin orientation between adjacent layers, resulted always in the total energy higher (or less favorable) than that of the FerriM state. However, this situation seen in finite number of multilayers (or thin films) changes in 3D periodic bulk $\mathrm{GdSi}_{2}$ as explained in the forthcoming section.

\section{3D bulk}

As described in Fig. 4(d) with $(1 \times 1 \times 2)$ supercell, 3D hexagonal $\mathrm{GdSi}_{2}$, gadolinium silicide crystal conforming with $P 6 / \mathrm{mmm}$ space group does exist in nature [38,39] and can be viewed as if it is built from the stacking of an infinite number of $2 \mathrm{D} \mathrm{GdSi} \mathrm{S}_{2}$ monolayers. The buckling of $\mathrm{Si}$ atoms existing in the monolayer, as well as in thin films, $2 \mathrm{~L}-4 \mathrm{~L}$, disappears, and the nearest $\mathrm{Si}-\mathrm{Si}$ distance is slightly shorter $(\sim 0.06 \AA)$ than that of the bulk Si. The spacing between Gd layers, $c=3.95 \AA$ is not too large and still allows chemical interaction. While the direction of the magnetic moments of adjacent Gd layers are antiparallel, their magnitudes have the same value, $\mu_{\mathrm{Gd}}=7.09 \mu_{B}$. Because of Abelian translation symmetry perpendicular to monolayers, even-odd disparity of layers disappears, but the antiparallel ordering of magnetic moments between adjacent layers is maintained to attribute an AFM ground state to 3D crystal. The FM configuration is an excited state with $38 \mathrm{meV}$ higher (unfavorable) energy. Accordingly, our results obtained from $\mathrm{PBE}+U+\mathrm{SOC}$ and HSE calculations finding an AFM ground state for 3D hexagonal gadolinium silicide are in agreement with experiment [2].

\section{SYMMETRIC STRUCTURES}

In view of the interesting properties of $\mathrm{Gd}_{2} \mathrm{~B}_{2}$ monolayer predicted recently by Gorkan et al. [12], we next consider $2 \mathrm{D} \mathrm{Gd}{ }_{2} \mathrm{Si}_{2}$ monolayer, where $\mathrm{Gd}$ atoms cover not only one side of silicene as in $2 \mathrm{D} \mathrm{GdSi}_{2}$ monolayer discussed in the previous sections, but also both sides to make a monolayer with a mirror symmetry as shown in Fig. 5(a), which has important implications.

\section{A. 2D Gd ${ }_{2} \mathrm{Si}_{2}$ monolayer}

Figure 5(a) describes the atomic structure and optimized values of relevant structure parameters. The spacing between Gd overlayers and Si plane is $1.85 \AA$; because of mirror symmetry the buckling of silicene between two Gd overlayers has disappeared leaving the nearest $\mathrm{Si}$-Si distance close to that of bulk Si crystal. This monolayer is found to be dynamically stable and maintain its stability above the room temperature. In Fig. 5(b), the calculated phonon bands are shown along with symmetry directions of the hexagonal BZ. The energy band structure calculated within $\mathrm{PBE}+U+\mathrm{SOC}$ is presented in Fig. 5(c). Spin-polarized bands calculated by HSE indicate an antiferromagnetic metal as shown in Fig. 5(d). Magnitudes of the magnetic moments of Gd atoms are located in the top and bottom Gd planes, $\mu_{\mathrm{Gd}}=7.26 \mu_{B}$ (HSE value is $7.29 \mu_{B}$ ); (a)

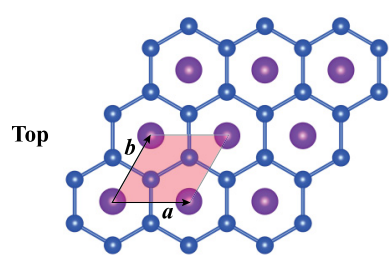

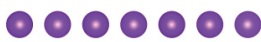

Side
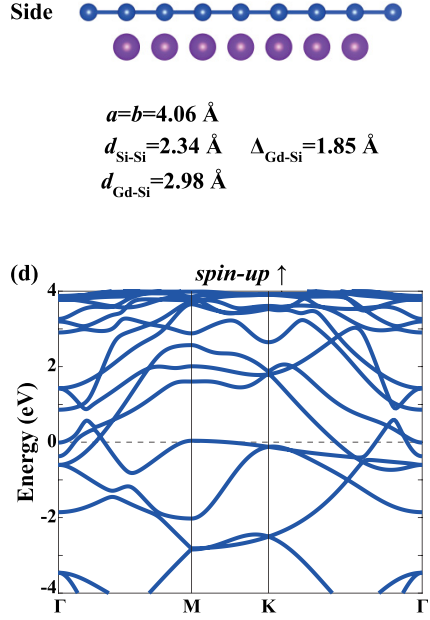

$$
\begin{aligned}
& a=b=4.06 \AA \\
& d_{\mathrm{Si}-\mathrm{Si}}=2.34 \AA \quad \Delta_{\mathrm{Gd}-\mathrm{Si}}=1.85 \AA \\
& d_{\mathrm{Gd}-\mathrm{Si}}=2.98 \AA
\end{aligned}
$$

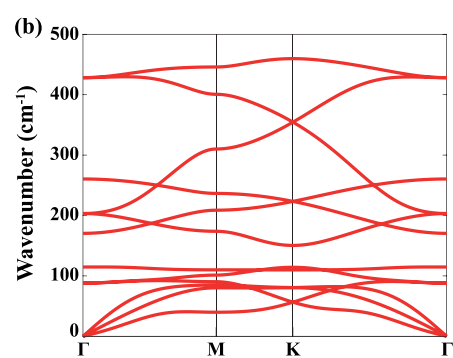

(c)

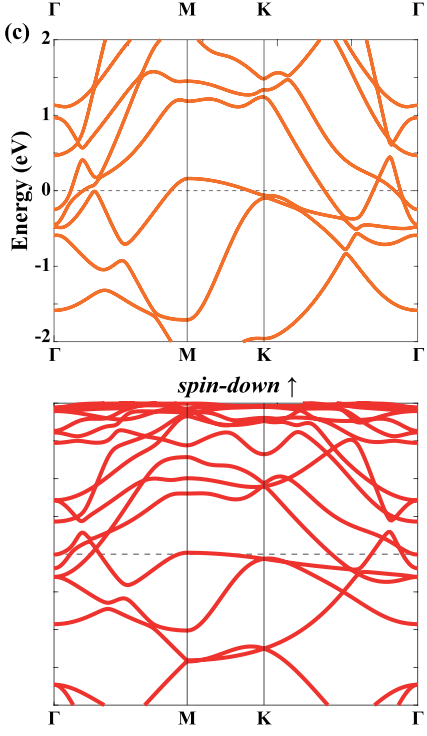

FIG. 5. (a) Top and side views of the atomic structure of $2 \mathrm{D}$ $\mathrm{Gd}_{2} \mathrm{Si}_{2}$ monolayers with the optimized values of relevant structural parameters. (b) Calculated phonon bands along with symmetry directions of hexagonal BZ. (c) Energy bands calculated by $\mathrm{PBE}+U+$ SOC. (d) Spin-polarized energy bands calculated by HSE.

but they are antiparallel to lead to AFM ground state. The antiferromagnetic ground state of $2 \mathrm{D} \mathrm{Gd}_{2} \mathrm{Si}_{2}$ monolayer is also tested using four different magnetic configurations in a rectangular supercell as shown in Fig. 6. Magnetic moments of these configurations indicate AFM ground state with $\mathrm{Gd}$ overlayers with antiparallel spin directions between two Gd layers.

\section{B. Multilayers with symmetric ordering of Gd overlayers}

To further investigate the effect of symmetry on the magnetic states of thin films constructed from $\mathrm{GdSi}_{2}$ monolayers, we now consider multilayers (thin films) with both sides covered by Gd overlayers. These symmetric multilayers are described in Fig. 7. Because of symmetry perpendicular to Gd layers, the multilayers with an even number of Gd layers have an AFM ground state; whereas those with an odd number of Gd layers have a FerriM ground state. None of these multilayers has an FM ground state, since the parallel ordering of magnetic moments in the same plane, but antiparallel ordering between nearest planes are favored. This is the dramatic difference between $\mathrm{GdSi}_{2}$ multilayers discussed in the foregoing section and the multilayers having mirror symmetry perpendicular to layers as shown in Fig. 7. Another interesting feature worth noting is that multilayers with an odd number of 

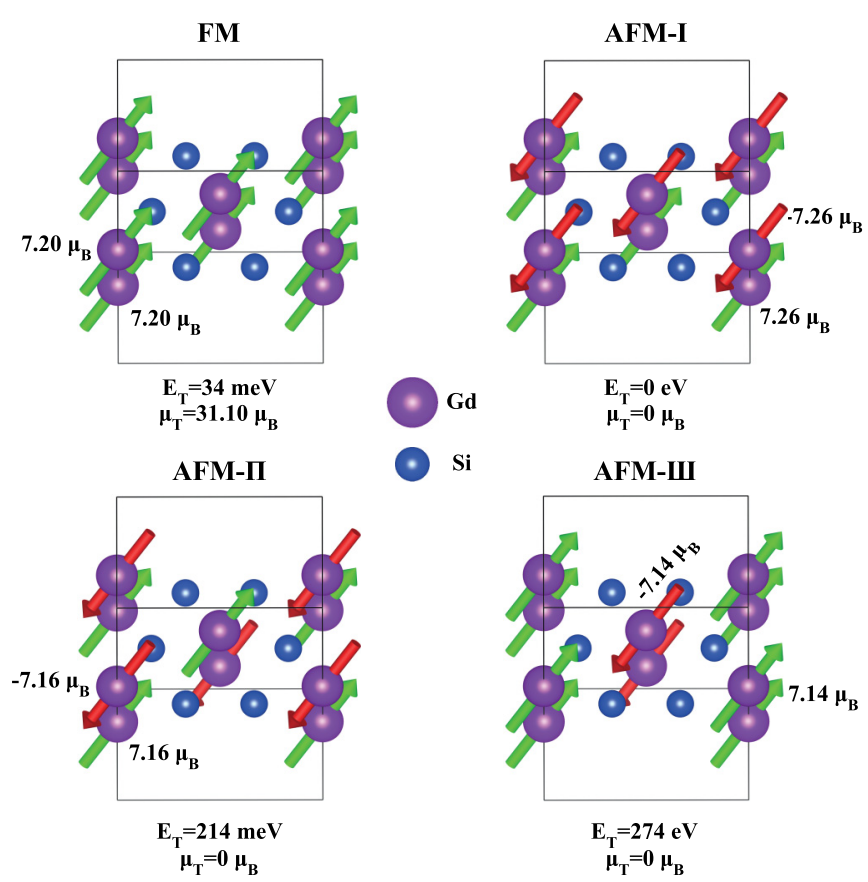

FIG. 6. Four different magnetic configurations, i.e., one ferromagnetic FM and three AFM (AFM-I, AFM-II, and AFM-III), and their calculated total energies. AFM-I configuration, where Gd atoms of the top and bottom overlayers have opposite spin directions with equal magnitudes, has the lowest total energy.

Gd planes have planar Si atomic planes, while those having an even number of Gd planes have slightly buckled Si layers.

\section{MONTE CARLO SIMULATIONS}

Monolayers of $\mathrm{GdSi}_{2}$ and $\mathrm{Gd}_{2} \mathrm{Si}_{2}$ discussed in the foregoing sections can be modeled by using the atomistic Hamiltonian

$$
\mathcal{H}=-J \sum_{\langle i j\rangle} \vec{\mu}_{i} \cdot \vec{\mu}_{j}-k_{x} \sum_{i}\left(\mu_{i}^{x}\right)^{2}-k_{y} \sum_{i}\left(\mu_{i}^{y}\right)^{2}
$$

where $\vec{\mu}_{i}$ is the classical spin vector with magnitude $\mu$ residing on the nodes of a square lattice with linear dimension $L=$ 100. The first summation is taken over the nearest neighbors, and the remaining summations are calculated over all lattice sites. $J$ and $k_{n}(n=x, y)$ respectively denote the exchange coupling and magnetic anisotropy constants. We employed the standard Metropolis method with a single site spin update scheme $[40,41]$ and applied periodic boundary conditions in each direction. In order to obtain statistically reasonable data, 100 different realizations were considered at each temperature. The simulation protocol is as follows: Initially, all spins are assigned random orientations corresponding to the disordered regime. Using this initial condition, we start the simulation at a temperature $T>T_{c}$ where $T_{c}$ is the critical temperature value separating the ordered and disordered phases. We gradually reduce the temperature in small steps down to $0.01 \mathrm{~K}$ which is assumed to correspond to the ground state configuration of the system. Various magnetic and thermodynamic properties of these monolayers were monitored over $10^{5}$ Monte Carlo steps per site after discarding the
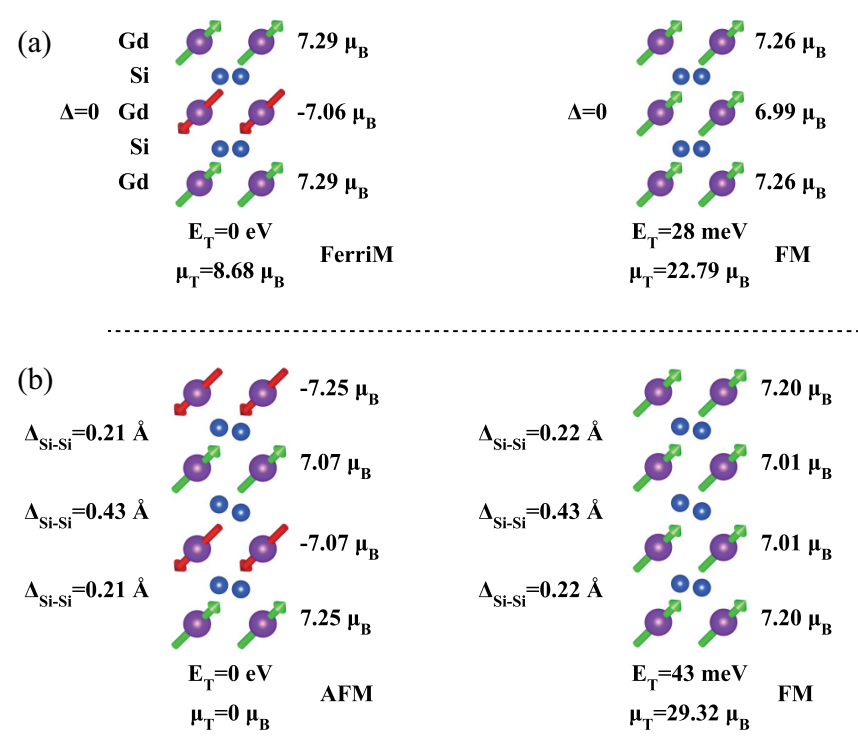

(c)
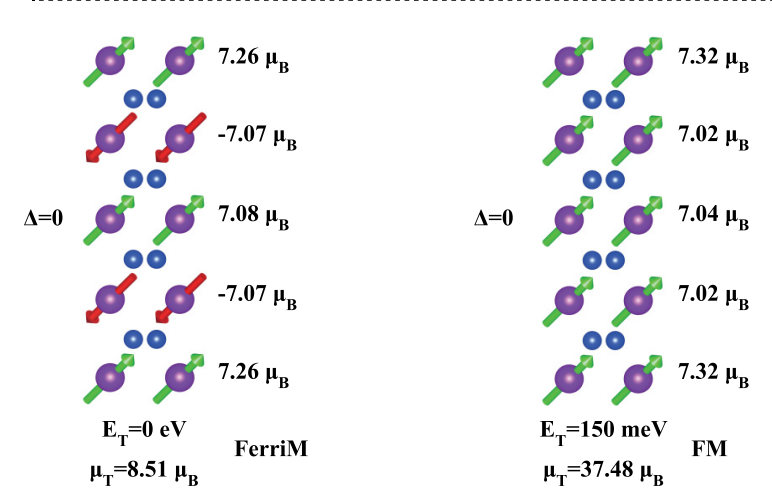

FIG. 7. Multilayers with the symmetric ordering of Gd overlayers, where the top and bottom sites are capped by Gd overlayers to acquire a mirror symmetry perpendicular to layers. (a) A multilayer including 3L Gd atoms with FerriM ground state and FM excited state. (b) A multilayer including 4L Gd atoms with AFM ground state and FM excited state. (c) A multilayer including 5L Gd atoms with FerriM ground state and excited FM state. The total energies, magnetic moments of $\mathrm{Gd}$ atoms, and buckling values of silicene layers $\Delta_{\mathrm{Si}-\mathrm{Si}}$ are indicated.

initial $10 \%$ of the data for the thermalization. These magnetic properties are FM magnetization expressed as

$$
\vec{M}=\frac{1}{N} \sum_{i=1}^{N} \vec{\mu}_{i},
$$

staggered AFM magnetization,

$$
\vec{M}_{S}=\frac{1}{N} \sum_{i=1}^{N}(-1)^{i} \vec{\mu}_{i},
$$

and the specific heat, as well as the magnetic susceptibility (FM and staggered) of the system which are respectively given by

$$
\begin{gathered}
C=d\langle\mathcal{H}\rangle / d T, \\
\chi_{O}=N\left(\left\langle\mathbf{O}^{2}\right\rangle-\langle\mathbf{O}\rangle^{2}\right) / k_{B} T,
\end{gathered}
$$



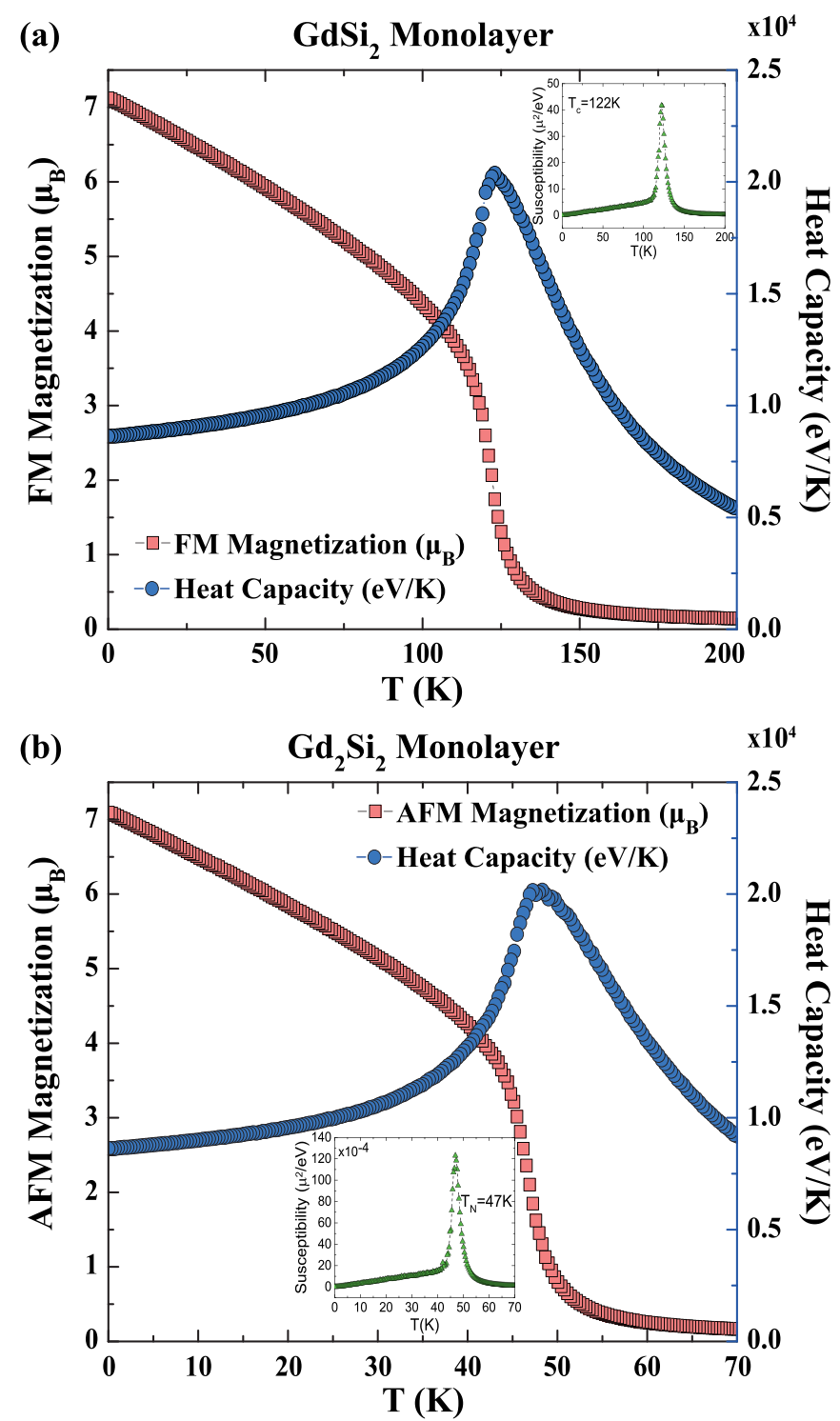

FIG. 8. Temperature dependence of (a) FM magnetization of $\mathrm{GdSi}_{2}$ monolayer and (b) AFM staggered magnetization of $\mathrm{Gd}_{2} \mathrm{Si}_{2}$ monolayer, each of which is accompanied by the corresponding heat capacity versus temperature curve. The insets show the variation of the magnetic susceptibility curves for each monolayer as functions of the temperature.

where $\mathbf{O}$ stands for the relevant order parameter vector of the system. In Eqs. (2) and (3), $N=L \times L$ refers to the total number of spins in the system.

Magnetic and thermodynamic properties of $\mathrm{GdSi}_{2}$ and $\mathrm{Gd}_{2} \mathrm{Si}_{2}$ monolayers revealed from Monte Carlo simulations are depicted in Fig. 8. As seen, the ground state configurations of $\mathrm{GdSi}_{2}$ and $\mathrm{Gd}_{2} \mathrm{Si}_{2}$ monolayers respectively correspond to FM and AFM formations, as were found by single- and double-cell DFT calculations. By inspecting the temperature dependencies of heat capacity curves, we find that the Curie temperature of $\mathrm{GdSi}_{2}$ monolayer is $T_{c}=122 \mathrm{~K}$, whereas the AFM Néel temperature $\mathrm{Gd}_{2} \mathrm{Si}_{2}$ monolayer is $T_{N}=47 \mathrm{~K}$. These results are also supported by temperature dependencies of magnetic susceptibility curves. It should be noted that
Tokmachev et al. [2] measured the Curie temperature of $\mathrm{GdSi}_{2}$ on $\mathrm{Si}(111)$ substrate $T_{c} \sim 40 \mathrm{~K}$, which is lower than that predicted by the present Monte Carlo simulations. This difference may be attributed to the fact that the $\mathrm{GdSi}_{2}$ monolayer in the experiment [2] was supported by $\mathrm{Si}(111)$ substrate, whereas $\mathrm{GdSi}_{2}$ monolayer considered in this study is freestanding.

\section{EFFECT OF STRAIN}

Earlier, several studies showed that magnetic properties of 2D monolayers can be monitored with applied compressive or tensile strain [12]. Here we first examined the effect of compressive, uniaxial and biaxial strain applied externally in the plane of $2 \mathrm{D} \mathrm{GdSi} i_{2}$ and $\mathrm{Gd}_{2} \mathrm{Si}_{2}$ monolayers in the range of $\pm 10 \%$ as summarized in Fig. 9. Calculations have been performed in the rectangular cell comprising two Gd atoms. No change in the magnetic ground state of $2 \mathrm{D} \mathrm{GdSi} i_{2}$ for the uniaxial strain along the $x$ axis, $\varepsilon_{x}$, and along the $y$ axis, $\varepsilon_{y}$, applied in the range $-10 \% \leqslant \varepsilon_{x, y} \leqslant 10 \%$. It is therefore inferred that $\mathrm{GdSi}_{2}$ monolayer continues to have FM ground state in this range of uniaxial applied strain. Variation of the magnetic moments on the Gd atom implies, however, the transition to AFM could be realized for larger strain values, where strain-induced structural instabilities might occur. In fact, under the biaxial tensile strain $\varepsilon_{x y}>9 \%$ the transition from FM to AFM ground state occurs.

However, 2D $\mathrm{Gd}_{2} \mathrm{Si}_{2}$ monolayer, which has an AFM ground state at $\varepsilon=0$, appears to be more sensitive to the applied strain. For $\varepsilon_{x}>9.5 \%$, this monolayer undergoes a magnetic transition and changes to FM ground state. The similar transition takes place for biaxial tensile strain already at $\varepsilon>4.5 \%$.

\section{DISCUSSIONS and CONCLUSIONS}

In spite of the fact that the nearest distance between $\mathrm{Gd}-\mathrm{Gd}$ atoms lying in the same plane ranges from $3.8 \AA$ to $4.0 \AA$ and those in the adjacent planes range from $3.70 \AA$ to $3.95 \AA$ in diverse gadolinium silicide structures, the present calculation is predicting always parallel orientation of magnetic moments in the same plane of $\mathrm{Gd}$ atoms, but antiparallel orientation between adjacent Gd planes. The physical mechanism underlying this intriguing order originates from the interplay of the diverse factors, like the nearest neighbor distance between $\mathrm{Gd}$ atoms located at the same and adjacent planes and also $\mathrm{Si}$ atoms (plane) intervening between $\mathrm{Gd}$ planes. In order to understand the order of magnetic moments and resulting magnetic ground states, we examined the role of $\mathrm{Si}$ atoms lying between adjacent $\mathrm{Gd}$ atomic planes. To this end, we considered 3D periodic $\mathrm{GdSi}_{2}$ with the planes of $\mathrm{Si}$ atoms in a honeycomb network. The AFM ground state of this crystal is constructed by the parallel magnetic moments of $\mathrm{Gd}$ atoms in the same plane, which are antiparallel to those in the nearest planes. To see the role of Si layers in the above magnetic interactions, we considered the $3 \mathrm{D} \mathrm{GdSi}_{2}$ with their original structural parameters, but all $\mathrm{Si}$ atoms are removed. This way any interaction involving $\mathrm{Si}$ atoms has been discarded, and only in-plane and interplane interactions between $\mathrm{Gd}$ atoms of comparable separation remain. Surprisingly, our self-consistent field (SCF) spin-polarized calculations using 

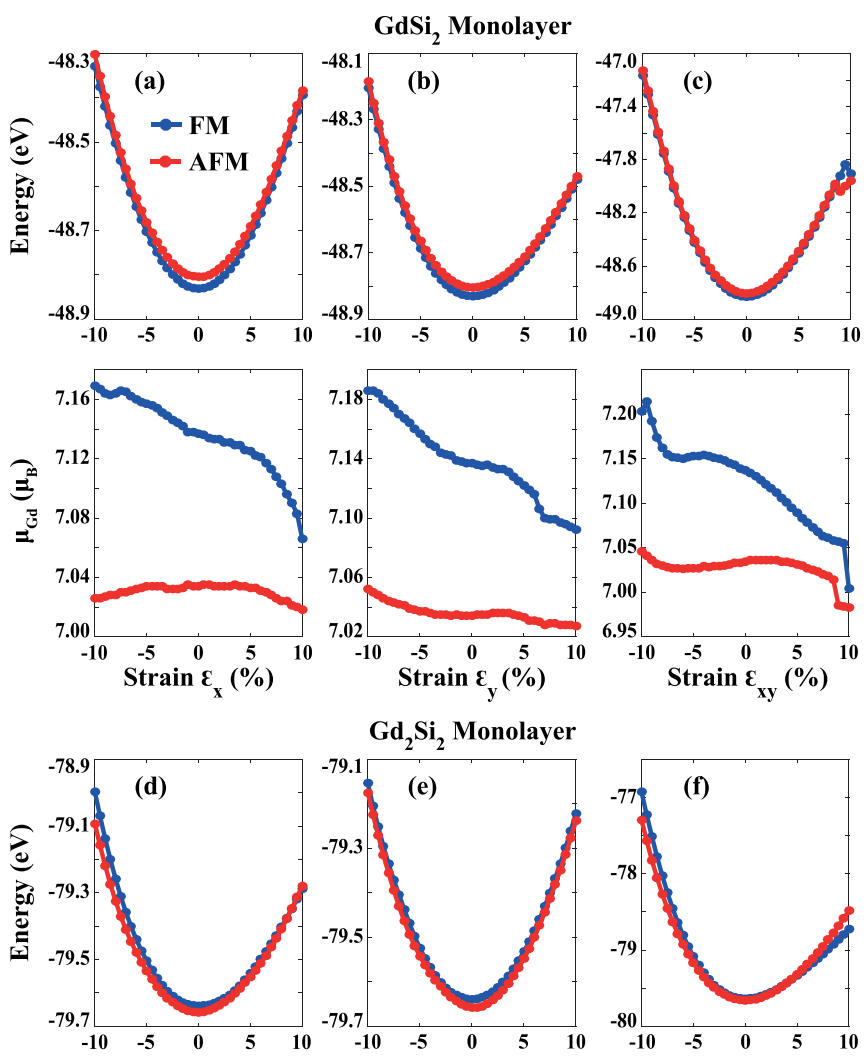

$\mathrm{Gd}_{2} \mathrm{Si}_{2}$ Monolayer
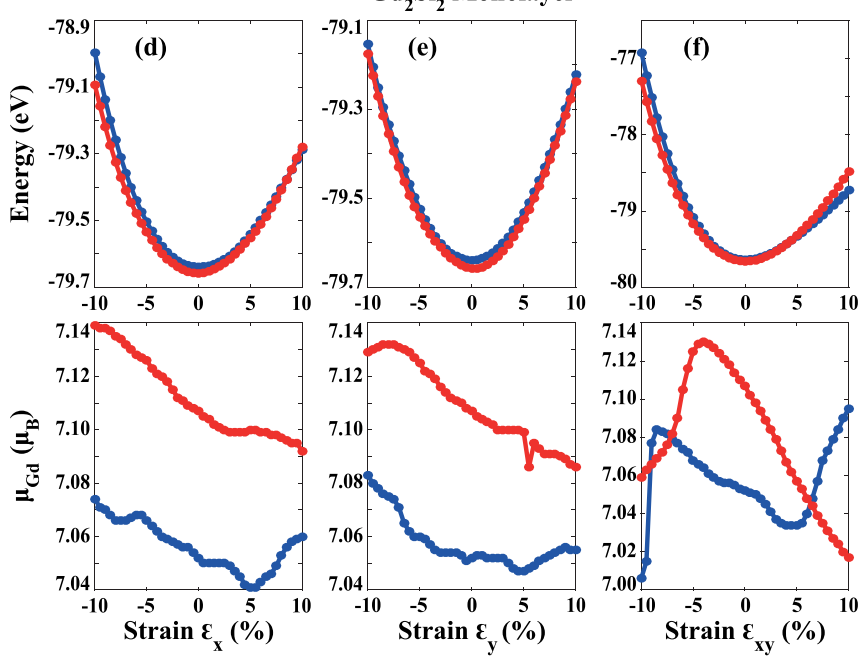

FIG. 9. Effect of the strain on the magnetic states and magnetic moments on $\mathrm{Gd}$ atoms of $2 \mathrm{D} \mathrm{GdSi}_{2}$ monolayer: (a) Top panel: Variation of the total energy. Bottom panel: magnetic moments on Gd atoms of FM and AFM magnetic states under uniaxial strain, $\varepsilon_{x}$. (b) Uniaxial strain $\varepsilon_{y}$. (c) Biaxial strain $\varepsilon_{x y}$. Effect of the strain on the magnetic states and magnetic moments on $\mathrm{Gd}$ atoms of $2 \mathrm{D} \mathrm{Gd}_{2} \mathrm{Si}_{2}$ monolayer: (d) Variation of the total energy and magnetic moment on Gd atoms of FM and AFM magnetic states under uniaxial strain $\varepsilon_{x}$. (e) Uniaxial strain $\varepsilon_{y}$. (f) Biaxial strain $\varepsilon_{x y}$.

$(2 \times 1 \times 2)$ and $(2 \times 2 \times 2)$ supercells of this hypothetical 3D Gd bulk resulted in the flip of magnetic moments in alternating Gd planes, so that all of them were aligned in the same direction to have strong FM ground state. This result suggests that $\mathrm{Si}$ atoms intervening between $\mathrm{Gd}$ planes force their magnetic moments to the antiparallel order. Thus, it is inferred that intervening $\mathrm{Si}$ atoms have a crucial effect on the magnetic states of 3D gadolinium silicide structures; a superexchangelike interaction through $\mathrm{Si}$ atoms keep the antiparallel spin alignment for all atoms between adjacent layers. This effect prevails also in all multilayers. $\mathrm{GdSi}_{2}$ monolayer, on the other hand, having only one Gd plane, is deprived from such an effect, and has magnetic moments of a single Gd plane that are aligned parallel to lead to FM ground state.

The present study of the effect of strain on the magnetic properties has been limited due to a narrow range of applied strain, since high strain values can give rise to structural instabilities. In this narrow range of applied strain, we found that the magnetic states of these materials are rather robust except a few magnetic phase changes can occur at specific strains. Nonetheless, interesting phase changes may occur at higher uniform 2D deformation and hydrostatic pressures. Similarly, we expect interesting magnetic configurations in different coverages such as $(2 \times 1),(2 \times 2)$, and ring structures, etc., whereby one can construct isolated magnetic wires (or chains) of $\mathrm{Gd}$ atoms on silicene with minute interchain coupling. Also, arrays of Gd vacancy or interstitial foreign atoms are expected to result in interesting magnetic structures.

In conclusion, the present theoretical paper studied stable atomic and electronic structures of $2 \mathrm{D} \mathrm{GdSi}_{2}$ monolayer, its multilayers, and $3 \mathrm{D}$ bulk together with $2 \mathrm{D} \mathrm{Gd}_{2} \mathrm{Si}_{2}$ monolayer, and provided for a consistent description of the prevailing magnetic interactions resulting in diverse magnetic states. In addition, the variation of magnetic states with symmetry, layer number, and strain were investigated. Recent experimental studies and present findings on gadolinium silicide demonstrate that 2D monolayers and atomically thin films constructed by the functionalization of monolayers of groupIV elements (such as graphene, silicene, germanene, stanene, their IV-IV and III-V compound monolayers in honeycomb structure, and transition metal dichalcogenides) by rare-earth atoms open a field for novel 2D spintronic nanomaterials.

\section{ACKNOWLEDGMENTS}

The computational resources are provided by TUBITAK ULAKBIM, High Performance and Grid Computing Center (TR-Grid e-Infrastructure). This work was supported by the Scientific and Technological Research Council of Turkey (TUBITAK) under Research Project No. 118F354. S.C. acknowledges the financial support of Academy of Science of Turkey (TÜBA). E.A. acknowledges support as a Humboldt Research Fellowship for Experienced Researchers.
[1] N. D. Mermim and H. Wagner, Absence of Ferromagnetism or Antiferromagnetism in One- or Two-Dimensional Isotropic Heisenberg Models, Phys. Rev. Lett. 17, 1133 (1966).

[2] A. M. Tokmachev, D. V. Averyanov, O. E. Parfenov, A. N. Taldenkov, I. A. Karateev, I. S. Sokolov, O. A. Kondratev, and
V. G. Storchak, Emerging two-dimensional ferromagnetism in silicene materials, Nat. Commun. 9, 1672 (2018).

[3] A. M. Tokmachev, D. V. Averyanov, A. N. Taldenkov, O. E. Parfenov. I. A. Karateev, I. S. Sokolov, and V. G. Storchak, Lanthanide $\mathrm{f}^{7}$ metalloxenes - a class of intrinsic 2D ferromagnets, Mater. Horiz. 6, 1488 (2019). 
[4] I. S. Sokolov, D. V. Averyanov, A. N. Taldenkov, O. E. Parfenov. I. A. Karateev, A. M. Tokmachev, and V. G. Storchak, 2D ferromagnetism in europium/graphene bilayers, Mater. Horiz. 7, 1372 (2020).

[5] K. S. Novoselov, A. K. Geim, S. V. Morozov, D. Jiang, Y. Zhang, S. V. Dubonos, I. V. Grigorieva, and A. A. Firsov, Electric field effect in atomically thin carbon films, Science 306, 666 (2004).

[6] E. Durgun, S. Tongay, and S. Ciraci, Silicon and III-V compound nanotubes: Structural and electronic properties, Phys. Rev. B 72, 075420 (2005).

[7] S. Cahangirov, M. Topsakal, E. Aktürk, H. Şahin, and S. Ciraci, Two- and One-Dimensional Honeycomb Structures of Silicon and Germanium, Phys. Rev. Lett. 102, 236804 (2009).

[8] S. Cahangirov, M. Topsakal, and S. Ciraci, Armchair nanoribbons of silicon and germanium honeycomb structures, Phys. Rev. B. 81, 195120 (2010).

[9] H. Şahin, S. Cahangirov, M. Topsakal, E. Bekaroglu, E. Aktürk, R. T. Senger, and S. Ciraci, Monolayer honeycomb structures of group-IV elements and III-V binary compounds: Firstprinciples calculations, Phys. Rev. B 80, 155453 (2009).

[10] P. Vogt, P. De Padova, C. Quaresima, J. Avila, E. Frantzeskakis, M. C. Asensio, A. Resta, B. Ealet, and G. Le Lay, Silicene: Compelling Experimental Evidence for Graphenelike TwoDimensional Silicon, Phys. Rev. Lett. 108, 155501 (2012)

[11] M. E. Davila, L. Xian, S. Cahangirov, A. Rubio, and G. L. Lay, Germanene: A novel two-dimensional germanium allotrope akin to graphene and silicene, New J. Phys. 16, 095002 (2014).

[12] T. Gorkan, E. Vatansever, Ü. Akıncı, G. Gokoglu, E. Aktürk, and S. Ciraci, Above room temperature ferromagnetism in $\mathrm{Gd}_{2} \mathrm{~B}_{2}$ monolayer with high magnetic anisotropy, J. Phys. Chem. C 124, 12816 (2020).

[13] F. Ersan, D. Kecik, V. O. Ozcelik, Y. Kadioglu, O. U. Akturk, E. Durgun, E. Akturk, and S. Ciraci, Two-dimensional pnictogens: A review of recent progresses and future research directions, Appl. Phys. Rev. 6, 021308 (2019).

[14] C. Gong and X. Zhang, Two-dimensional magnetic crystals and emergent heterostructure devices, Science 363, eaav4450 (2019).

[15] K. S. Burch, D. Madrus, and J. G. Park, Magnetism in twodimensional van der Waals materials, Nature (London) 563, 47 (2018).

[16] M. Gibertini, M. Koperski, A. F. Morpurgo, and K. S. Novoselov, Magnetic 2D materials and heterostructures, Nat. Nanotechnol. 14, 408 (2019).

[17] B. Huang, G. Clark, E. Navarro-Moratalla, D. R. Klein, R. Cheng, K. L. Seyler, D. Zhong, E. Schmidgall, M. A. McGuire, D. H. Cobden et al., Layer-dependent ferromagnetism in a van der Waals crystal down to the monolayer limit, Nature (London) 546, 270 (2017).

[18] C. Gong, L. Li, Z. Li, H. Ji, A. Stern, Y. Xia, T. Cao, W. Bao, C. Wang, Y. Wang et al., Discovery of intrinsic ferromagnetism in two-dimensional van der Waals crystals, Nature (London) 546, 265 (2017).

[19] B. Huang, G. Clark, D. R. Klein, D. MacNeill, E. NavarroMoratalla, K. L. Seyler, N. Wilson, M. A. McGuire, D. H. Cobden, D. Xiao et al., Electrical control of 2D magnetism in bilayer $\mathrm{CrI}_{3}$, Nat. Nanotechnol. 13, 544 (2018).

[20] X. Zhenjia (C. C. Hsu), in Properties of Metal Silicides, edited by K. Maex, and M. van Rossum (INSPEC, London, 1995), p. 45 .
[21] P. E. Blöchl, Projector augmented-wave method, Phys. Rev. B 50, 17953 (1994).

[22] G. Kresse, and D. Joubert, From ultrasoft pseudopotentials to the projector augmented-wave method, Phys. Rev. B 59, 1758 (1999).

[23] G. Kresse and J. Furthmüller, Efficient iterative schemes for $a b$ initio total-energy calculations using a plane-wave basis set, Phys. Rev. B 54, 11169 (1996).

[24] J. P. Perdew, K. Burke, and M. Ernzerhof, Generalized Gradient Approximation Made Simple, Phys. Rev. Lett. 77, 3865 (1996).

[25] S. L. Dudarev, G. A. Botton, S. Y. Savrasov, C. J. Humphreys, and A. P. Sutton, Electron-energy-loss spectra and the structural stability of nickel oxide: An LSDA1U study Phys. Rev. B 57, 1505 (1998).

[26] L. Oroszlány, A. Deák, E. Simon, S. Khmelevskyi, and L. Szunyogh, Magnetism of Gadolinium: A First-Principles Perspective, Phys. Rev. Lett. 115, 096402 (2015).

[27] V. I. Anisimov, F. Aryasetiawan, and A. I. Lichtenstein, Firstprinciples calculations of the electronic structure and spectra of strongly correlated systems: The LDA $+U$ method, J. Phys.: Condens. Matter 9, 767 (1997).

[28] I. Turek, J. Kudrnovský, G. Bihlmayer, and S. Blügel, Ab initio theory of exchange interactions and the Curie temperature of bulk Gd, J. Phys.: Condens. Matter 15, 2771 (2003).

[29] S. Grimme, Semiempirical GGA-type density functional constructed with a long-range dispersion correction, J. Comput. Chem. 27, 1787 (2006).

[30] H. J. Monkhorst and J. D. Pack, Special points for Brillouinzone integrations, Phys. Rev. B 13, 5188 (1976).

[31] C. G. Broyden, The convergence of a class of double-rank minimization algorithms: 1. General considerations, IMA J. Appl. Math. 6, 76 (1970).

[32] C. G. Broyden, The convergence of a class of double-rank minimization algorithms: 2. The new algorithm, IMA J. Appl. Math. 6, 222 (1970).

[33] S. Baroni, S. de Gironcoli, A. Dal Corso, and P. Giannozzi, Phonons and related crystal properties from density-functional perturbation theory, Rev. Mod. Phys. 73, 515 (2001).

[34] A. Togo and I. Tanaka, First principles phonon calculations in materials science, Scr. Mater. 108, 1 (2015).

[35] K. Momma and F. Izumi, VESTA 3 for three-dimensional visualization of crystal, volumetric and morphology data, J. Appl. Crystallogr. 44, 1272 (2011).

[36] A. V. Krukau, O. A. Vydrov, A. F. Izmaylov, and G. E. Scuseria, Influence of the exchange screening parameter on the performance of screened hybrid functionals, J. Chem. Phys. 125, 224106 (2006).

[37] M. Aras, C. Kilic, and S. Ciraci, Magnetic ground state in $\mathrm{FeTe}_{2}, \mathrm{VS}_{2}$, and $\mathrm{NiTe}_{2}$ monolayers: Antiparallel magnetic moments in chalcogen atoms, Phys. Rev. B 101, 054429 (2020).

[38] V. M. Koleshko, V. F. Belitoky, and A. A. Khodin, Thin films of rare earth metal silicides, Thin Solid Films 141, 277 (1986)

[39] J. A. Knapp and S. T. Picraux, Epitaxial growth of rare-earth silicides on (111) Si, Appl. Phys. Lett. 48, 466 (1986).

[40] K. Binder and D. Landau, A Guide to Monte Carlo Simulations in Statistical Physics (Cambridge University Press, Cambridge, 2009).

[41] M. E. J. Newman and G. T. Barkema, Monte Carlo Methods in Statistical Physics (Oxford University Press, New York, 2001). 